\title{
Fabrication of Mesoporous Carbon Materials from Non-mesoporous Silica Spheres Templates
}

\author{
Jae Kwang Lee, Yong-Ki Park, Kyu Yong Choi, Hee Young Kim, and Chul Wee Lee* \\ Advanced Chemical Technology Division, Korea Research Institute of Chemical Technology, Daejeon 305-600, Korea \\ *E-mail: chulwee@krict.re.kr \\ Received September 17, 2004
}

Key Words : Nano carbon ball, Mesoporous carbon

Recently, many workers have been investigated the possibility of fabricating various replicas from inorganic templates. ${ }^{1,2}$ Based on these investigations, it is anticipated that nano-sized carbon capsules would constitute excellent materials for many fields of research and many different industries. ${ }^{3,4}$ It is well known that shrinkage and cracking are induced in carbon materials during the carbonization or pyrolysis processes, ${ }^{5,7}$ and it can be assumed that the such phenomena are sufficient to create mesopores, if the thickness of the carbonizing material is very thin, such as in the case of nano-sized carbon shell.

In this study, we attempted to fabricate mesoporous carbon hollow spheres (hereinafter, referred to as CHSs) from nonmesoporous silica spheres through their carbonization and activation, and simultaneously estimate the difference in the physical properties between two types of CHSs prepared from phenol-formaldehyde resins (hereinafter, PF-resin) synthesized in either the gas or liquid phase. Herein, the synthesis in the liquid phase was employed for the purpose of preparing thicker carbon shells than in the gas phase.

Silica spheres with diameters in the range of 450-500 nm were synthesized according to the sol-gel method. ${ }^{6}$ The terms CHS-gas and CHS-liquid are used to indicate the carbon hollow spheres fabricated in the gas and liquid phases, respectively, while AcCHSs refers to the activated carbon hollow spheres. The SEM and TEM images of the CHSs are shown in Figure 1. The CHS-gas spheres shown in Figure 1(A) had an average core diameter of $500 \mathrm{~nm}$ and an average shell thickness of about $10 \mathrm{~nm}$. The CHS-liquid spheres shown in Figure 1(B) had an average core diameter of $500 \mathrm{~nm}$ and an average shell thickness of about 40-50 nm.

The adsorption/desorption isotherms and $\mathrm{BJH}$ pore distributions of the various CHSs are shown in Figure 2. In the case of the spheres synthesized in the gas phase, the isotherms of both the CHS-gas and AcCHS-gas spheres were designated as type $\mathrm{H} 3$, as recommended in the IUPAC manual, indicating that their carbon shells consisted with almost plate-like particles. ${ }^{8}$ Both the isotherm and pore distribution of the AcCHS-gas spheres had similar patterns to those of the CHS-gas spheres, indicating that the activation process served to improve their pore volume, in particular due to the generation mesopores with a diameter of 2-4 $\mathrm{nm}$, that were created during the carbonization process. And the pore volume above $10 \mathrm{~nm}$ could be
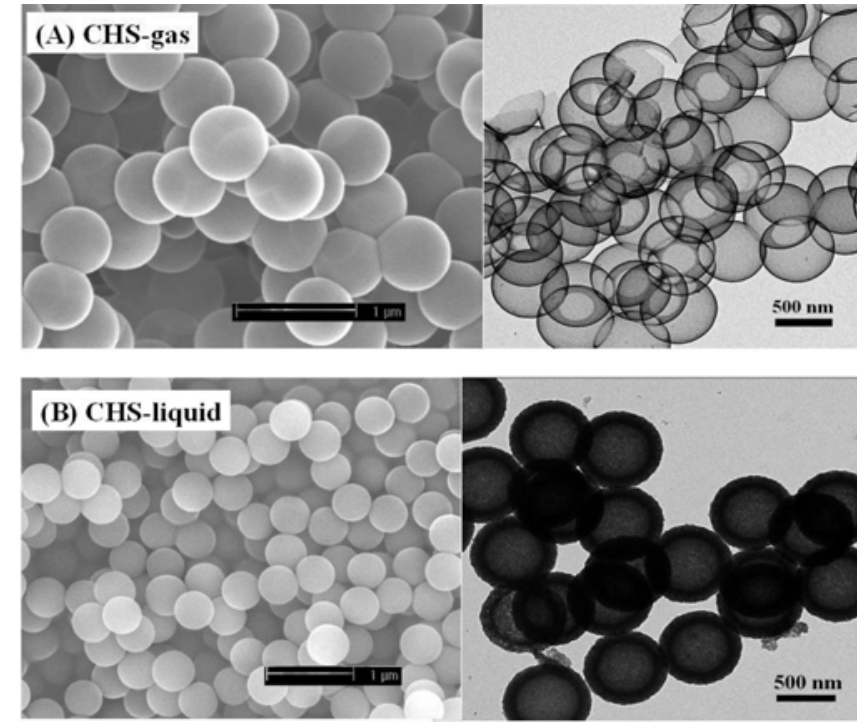

Figure 1. SEM and TEM images of the various CHSs [(A) CHSgas, (B) CHS-liquid].

attributed to the bulk pores between those spheres because of the thin and flexible shells. In the case of the spheres synthesized in the liquid phase, the isotherms of both the CHS-liquid and AcCHS-liquid spheres were designated as type H3. However, the pattern of the AcCHS-liquid spheres was not similar to that of the CHS-liquid spheres at a relative pressure of around 0.5, and was distinctly different from those of the above-mentioned CHS-gas and AcCHS-gas spheres. This can be attributed to the pore distributions of both the CHS-liquid and AcCHS-liquid spheres synthesized in the liquid phase. The pore volume of the CHS-liquid spheres increased with decreasing pore diameter, whereas the pore volume of the AcCHS-liquid spheres remarkably increased within the range of pore diameters of about 3-4 $\mathrm{nm}$, indicating that the activation of the carbonized AlSiRliquid spheres increased the number of mesopores generated from the micropores created during the carbonization process. However, this abrupt variation could not be considered to result merely within the increased number of mesopore from the micropores, following two reasons; firstly, because the total pore volume of the CHS-liquid spheres, which has a value of $0.65 \mathrm{~cm}^{3} / \mathrm{g}$ calculated by the BJH method, is less than that of the AcCHS-liquid spheres, whose value is 1.3 

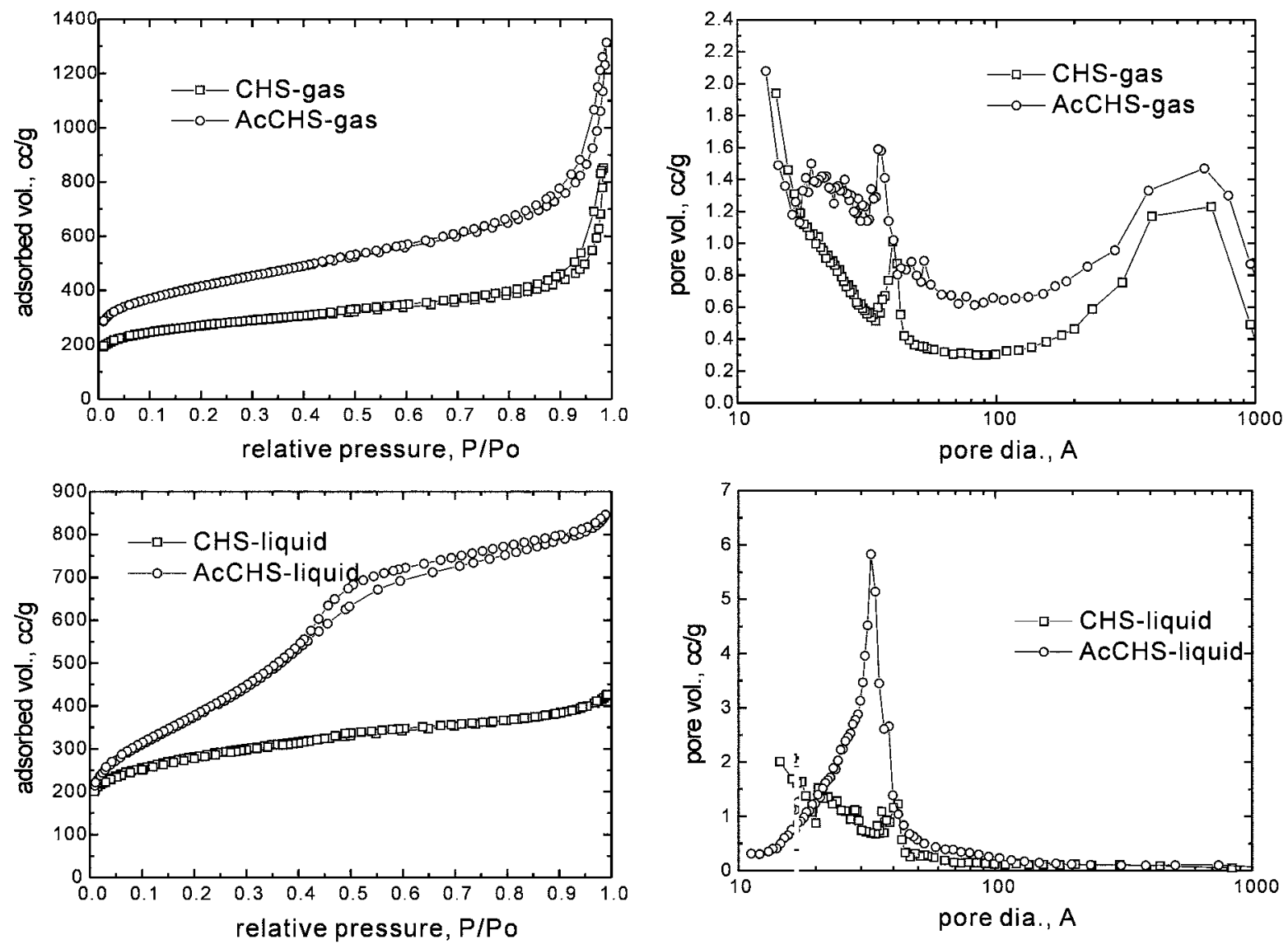

Figure 2. Nitrogen adsorption/desorption isotherms and BJH pore distributions of the various CHSs.

Table 1. Physical properties of the various CHSs from the BET measurements

\begin{tabular}{lccccc}
\hline Properties & Unit & $\begin{array}{c}\text { CHS- } \\
\text { gas }\end{array}$ & $\begin{array}{c}\text { AcCHS- } \\
\text { gas }\end{array}$ & $\begin{array}{c}\text { CHS- } \\
\text { liquid }\end{array}$ & $\begin{array}{c}\text { AcCHS- } \\
\text { liquid }\end{array}$ \\
\hline Surface area & {$\left[\mathrm{m}^{2} / \mathrm{g}\right]$} & 958 & 1464 & 999 & 1360 \\
$\begin{array}{l}\text { Total pore volume } \\
{\left[\mathrm{cm}^{3} / \mathrm{g}\right]}\end{array}$ & 1.3 & 2 & 0.65 & 1.3 \\
$\begin{array}{l}\text { Average pore } \\
\text { diameter }\end{array}$ & {$[\mathrm{nm}]$} & 5.5 & 5.5 & 2.6 & 3.8 \\
\hline
\end{tabular}

$\mathrm{cm}^{3} / \mathrm{g}$, and secondly, because non-micropores are created during the activation process. Therefore, it can be assumed that the activation process may open mesopores, which were created and subsequently clogged during the carbonization of the AISiR spheres synthesized in the liquid phase.

The physical properties inferred from the isotherms are listed in Table 1. The total pore volumes of all of the CHSs are higher than that of commercial activated carbon, for which the value is in the range of $0.4-0.6 \mathrm{~cm}^{3} / \mathrm{g}$, regardless of the surface areas. In addition, although the properties of the CHSs synthesized in the gas phase are better than those of the CHSs synthesized in the liquid phase, considering the pore distributions shown in Figure 2, the number of mesopores with diameters of 3-4 $\mathrm{nm}$ is abruptly increased by the activation of the carbonized AlSiR synthesized in the liquid phase.

From these results, we propose that mesopores can be created on the carbon shells of carbon hollow spheres using PF-resin coated aluminosilicate spheres as a non-mesoporous template. In addition, the water vapor activation of the carbonized aluminosilicate resin synthesized in the liquid phase provides a very effective means of fabricating these mesoporous carbon hollow spheres with a higher volume of mesopores having a specific size, in the range of 3-4 $\mathrm{nm}$.

Acknowledgement. This work was supported by the Ministry of Science and Technology of Korea and LG Household \& Healthcare Research Park.

\section{References}

1. Kwon, O.-Y.; Park, K.-W. Bull. Korean Chem. Soc. 2003, 24 , 1561.

2. Cheon, J. H.; Kim, S. J.; Choe, K. J.; Park, D. H. Bull. Korean Chem. Soc. 2004, 25, 21.

3. Lee, J. W.; Sohn, K. N.; Hyeon, T. W. J. Am. Chem. Soc. 2001, $123,5146$.

4. Yoon, S. B.; Sohn, K. N.; Kim, J. Y.; Shin, C. H.; Yu, J. S.; Hyeon, T. W. Adv. Mater. 2002, 14, 19.

5. Donnet, J. B.; Bansal, R. C. CARBON FIBERS; Marcel Dekker, Inc.: New York, 1990; $\mathrm{p} 281$

6. Vacassy, R.; Flatt, R. J.; Hofmann, H.; Choi, K. S.; Singh, R. K. Journal of Colloid and Interface Science 2000, 227, 302.

7. Worasuwannarak, N.; Hatori, S.; Nakagawa, H.; Miura, K. Carbon 2003, 41, 933.

8. Gregg, S. J.; Sing, K. S. W. Adsorption, Surface Area and Porosity; Academic Press: London, 1982; p 283. 